\title{
Numerical and experimental study of fluid flow in simplified blade cascade with prescribed harmonic motion
}

\author{
Jan Vimmr ${ }^{1, \star}$, Ondřej Bublík ${ }^{1}$, Aleš Pecka ${ }^{1}$, Luděk Pešek ${ }^{2, \star \star}$, and Pavel Procházka ${ }^{2}$ \\ ${ }^{1}$ NTIS, Faculty of Applied Sciences, University of West Bohemia, Pilsen, Czech Republic \\ ${ }^{2}$ Institute of Thermomechanics AS CR, v.v.i., Academy of Sciences of the Czech Republic, Prague, Czech Republic
}

\begin{abstract}
In this paper, a numerical simulation and experimental measurements of compressible viscous fluid flow in simplified blade cascade are compared. The cascade consists of five flat plates three of which perform prescribed harmonic motion. The computed unsteady velocity field is compared with experimental measurements at selected points. Moreover, the power spectral density corresponding to the frequency of harmonic motion is computed for both the numerical and experimental data and compared. The numerical simulation was performed using the developed in-house CFD software based on the discontinuous Galerkin method, which offers high order of accuracy.
\end{abstract}

\section{Introduction}

The first papers focusing on the aero-elasticity of blades in axial turbines appeared in the forties of the 20th century [1], [2]. Here an empirical criterion for flutter occurrence was introduced. It is based on a reduced blade frequency for bending blade vibration. This criterion however neglects other influences, such as geometric configuration of blade cascade or angle of attack, which may also lead to flutter. Nowadays the problem of aero-elasticity is still a subject of topical interest because modern turbines are designed for higher efficiencies and higher power under higher operational temperatures and flow rates. Higher operational, safety and economical demands force the designers to be more precise during the process of determination of safe operational condition laying out of the area with loss of stability.

In the work [3] it has been shown that the critical flutter point, i.e. the highest aero-dynamical excitation, arises for a particular inter-blade phase angle. Most of aerodynamic studies are based on the so called travelling wave mode of vibration, where one assumes that all blades vibrate at the same frequency, amplitude and inter-blade phase angle. Other aerodynamic studies use the influence coefficient technique, which is based on the calculation of aerodynamic forces of vibrating blade acting on non-vibrating adjacent blades.

In our previous work [4] we used the travelling wave mode approach for the numerical modelling of the unsteady flow in the simplified blade cascade formed by three flat plates. We prescribed a harmonic motion for all three flat plates in the cascade such that their mutual movement produces a travelling wave. The flutter instability occurred for specific phase angles as expected.

In this study, we use the same concept of the travelling wave mode approach. In order for the configuration to correspond to a real blade disc better, we have added two fixed plates on the top and the bottom. The experiments have also been carried out for the same configuration. The numerical and experimental data were compared in both the steady case, where all of the plates are fixed in time, and for the transient case, where the three middle plates perform a harmonic motion and the two outermost blades are still.

The numerical simulations are based on the solution of the two-dimensional system of Favre-averaged NavierStokes equations, in dimensionless arbitrary LagrangianEulerian (ALE) form. The computations were performed using the developed in-house CFD software FlowPro based on the discontinuous Galerkin finite element method.

\footnotetext{
^e-mail: jvimmr@kme.zcu.cz

$\star \star$ e-mail: pesek@it.cas.cz
} 


\section{Mathematical model}

The two-dimensional problem of compressible viscous fluid flow through simplified blade cascade with prescribed harmonic motion is described by Favre-averaged system of Navier-Stokes equations, in dimensionless arbitrary Lagrangian-Eulerian (ALE) formulation written as

$$
\begin{aligned}
& \frac{D^{A} \bar{\varrho}}{D t}+\frac{\partial\left(\bar{\varrho} \tilde{u}_{j}\right)}{\partial x_{j}}-U_{j} \frac{\partial \bar{\varrho}}{\partial x_{j}}=0, \\
& \frac{D^{A}}{D t}\left(\bar{\varrho} \tilde{u}_{i}\right)+\frac{\partial}{\partial x_{j}}\left(\bar{\varrho} \tilde{u}_{i} \tilde{u}_{j}+\bar{p} \delta_{i j}\right)-U_{j} \frac{\partial\left(\varrho \tilde{\varrho}_{i}\right)}{\partial x_{j}}= \\
& \qquad \frac{1}{\operatorname{Re} \frac{\partial}{\partial x_{j}}\left(\tilde{t}_{i j}+\tau_{i j}\right),} \\
& \frac{D^{A}}{D t}(\bar{\varrho} \tilde{e})+\frac{\partial}{\partial x_{j}}\left(\bar{\varrho} \tilde{e} \tilde{u}_{j}+\bar{p} \tilde{u}_{j}\right)-U_{j} \frac{\partial(\bar{\varrho} \tilde{e})}{\partial x_{j}}= \\
& \frac{1}{\operatorname{Re}} \frac{\partial}{\partial x_{j}}\left[\left(\tilde{t}_{i j}+\tau_{i j}\right) \tilde{u}_{i}+\frac{\kappa}{\kappa-1}\left(\frac{\mu}{P r}+\frac{\mu_{t}}{P r_{t}}\right) \frac{\partial}{\partial x_{j}}\left(\frac{\bar{p}}{\bar{\varrho}}\right)\right],
\end{aligned}
$$

where $i, j=1,2, \bar{\varrho}$ and $\bar{p}$ is the dimensionless timeaveraged density and pressure, $\tilde{u}_{i}$ and $\tilde{e}$ is the dimensionless mass-averaged velocity component and energy, $R e$ is the Reynolds number, $\operatorname{Pr}$ and $\operatorname{Pr}_{t}$ is the Prandtl number and the turbulent Prandtl number, $\mu$ and $\mu_{t}$ is the dynamic and turbulent viscosity and $U_{j}$ is the $\mathrm{j}$-th component of mesh velocity. For a flow around flat plates, the value of the turbulent Prandtl number is $P r_{t}=0.89$. Mass-averaged stress tensor and Reynolds stress tensor are given by

$$
\tilde{t}_{i j}=2 \mu \bar{S}_{i j}, \tau_{i j}=2 \mu_{t} \bar{S}_{i j}
$$

where

$$
\bar{S}_{i j}=\frac{1}{2}\left(\frac{\partial \tilde{u}_{i}}{\partial x_{j}}+\frac{\partial \tilde{u}_{j}}{\partial x_{i}}\right)-\frac{1}{3} \delta_{i j} \frac{\partial \tilde{u}_{k}}{\partial x_{k}} .
$$

The equation of state for ideal gas is considered, i.e.

$$
\bar{p}=(\kappa-1)\left[\bar{\varrho} \tilde{e}-\frac{1}{2} \bar{\varrho} \sum_{i=1}^{2} \tilde{u}_{i}^{2}\right] \text {. }
$$

Finally the symbol $\frac{D^{A}}{D t}$ denotes the ALE derivative [5].

To include the influence of turbulent fluctuations on the mean flow a one-equation turbulence model of Spalart and Allmaras [6] is used. It is given by the transport equation for eddy viscosity $\tilde{v}$ written as

$$
\begin{gathered}
\frac{D^{A}(\bar{\varrho} \tilde{v})}{D t}+\frac{\partial\left(\bar{\varrho} \tilde{v} \tilde{u}_{j}\right)}{\partial x_{j}}-U_{j} \frac{(\partial \bar{\varrho} \tilde{v})}{\partial x_{j}}= \\
\frac{1}{\operatorname{Re}} \frac{1}{\sigma}\left[\frac{\partial}{\partial x_{j}}\left((\mu+\bar{\varrho} \tilde{v}) \frac{\partial \tilde{v}}{\partial x_{j}}\right)+\bar{\varrho} c_{b 2} \frac{\partial \tilde{v}}{\partial x_{j}} \frac{\partial \tilde{v}}{\partial x_{j}}\right]+ \\
c_{b 1} \bar{\varrho} \tilde{S} \tilde{v}-\frac{1}{R e} c_{w 1} f_{w} \frac{1}{\bar{\varrho}}\left(\frac{\bar{\varrho} \tilde{v}}{D}\right)^{2}
\end{gathered}
$$

and completed by the following relations

$$
\mu_{t}=\varrho \tilde{\varrho} \tilde{v} f_{v 1},
$$

$$
\begin{aligned}
& \tilde{S}=\bar{\Omega}+\frac{1}{\operatorname{Re}} \frac{\tilde{v}}{\kappa^{2} D^{2}} f_{v 2}, \\
& f_{w}=g\left(\frac{1+c_{w 3}^{6}}{g^{6}+c_{w 3}^{6}}\right)^{\frac{1}{6}}, \\
& g=r+c_{w 2}\left(r^{6}-r\right), \\
& r=\min \left(\frac{\tilde{v}}{\operatorname{Re} \tilde{S} \kappa^{2} D^{2}}, 10\right), \\
& f_{v 1}=\frac{\chi^{3}}{\chi^{3}+c_{v 1}^{3}}, \quad \chi=\frac{\tilde{v}}{v}, \\
& f_{v 2}=1-\frac{\chi}{1+\chi f_{v 1}},
\end{aligned}
$$

where $D$ is the distance to the nearest wall and $\bar{\Omega}$ is the vorticity magnitude. The constants for the turbulence model are $c_{b 1}=0.1355, c_{b 2}=0.622, \sigma=\frac{2}{3}, \kappa=0.41, c_{w 1}=$ $\frac{c_{b 1}}{\kappa^{2}}+\frac{1+c_{b 2}}{\sigma}, c_{w 2}=0.3, c_{w 3}=2.0, c_{v 1}=7.1$,

The discretization of the mathematical model in ALE formulation using the discontinuous Galerkin method [7], [8] is described in previous works [4] and [9] in detail. Here, a simple algorithm for mesh deformation is also described. The mentioned mathematical model is incorporated in the developed inhouse numerical software called FlowPro. The software is validated on an inviscid compressible unsteady fluid flow around oscillating NACA0012 airfoil for a free stream Mach number $M_{\infty}=$ 0.755 [4]. The instantaneous motion of the airfoil is given as a function of time

$$
\alpha(t)=\alpha_{m}+\alpha_{o} \sin (\omega t)
$$

which defines the angle of tilt of the airfoil. Here the mean angle $\alpha_{m}=0.016^{\circ}$ and the amplitude of oscillation $\alpha_{o}=2.51^{\circ}$. The angular velocity is defined as $\omega=2 k u_{\infty} / c$, where reduced frequency $k=0.0814$, profile chord length $c=1$ and $u_{\infty}$ is the free-stream velocity. The coordinates of the reference point of rotation are $x_{r e f}=[0.25,0]$. Figure 1 shows a comparison of obtained numerical results (red) by the developed code with the results published in [10] and [11] (black), which are computed by finite volume solvers. A reasonably good agreement is apparent from both of the test problems, which confirms a correct implementation of the implicit DGFEM solver along with the ALE approach.

\section{Experimental setup}

The simplified blade cascade was chosen for the study of flutter in order to achieve a simple experimental setup. The experimental setup that was already constructed at the Institute of Thermomechanics AS CR is described in this section. The experimental stand of the simplified blade cascade is shown in Figure 2. The cascade consists of 5 flat plates numbered 2, 1, 0, -1 and -2 from top to bottom. Each plate is on one of its ends attached to the base by four spring steel belts. The outermost plates -2 and 2 are steady, thus have zero degrees of freedom and are present in order to simulate the periodic boundary condition. The three middle plates $-1,0$ and 1 can move only in the horizontal 

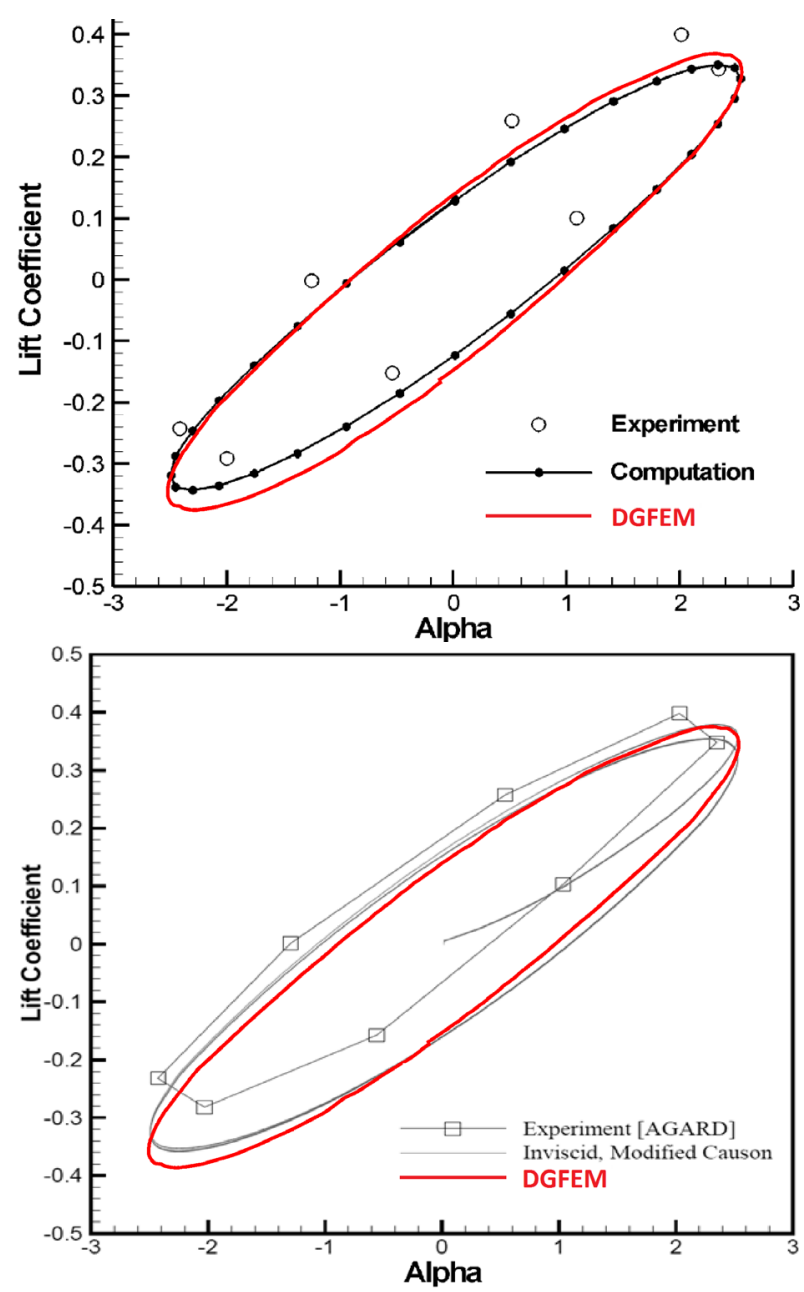

Fig. 1. Dependence of the lift coefficient $c_{L}$ on the angle of the airfoil. Comparison with the numerical and experimental results published in [10] (top) and [11] (bottom).

direction, hence the simplified blade cascade has three degrees of freedom. The harmonic motion with prescribed amplitude, frequency and phase of the three middle plates is accomplished by electrodynamic exciters, which are attached to the base through two L-shaped beams and linked to the plates by a rod. A force transducer, which records inertial and elastic forces of the vibrating plate and aerodynamic forces of the fluid, is placed at the end of each rod. The movement of the plate is recorded by the foil strain gauges, which are calibrated for the displacement of the plates. Two strain gauges are glued onto both sides of each spring steel belt and they are connected to the semibridge circuit. The incidental angle of the flow and the cascade can be changed by rotating the whole base.

\section{Results}

In this section, the experimental data are compared with the numerical results computed by the developed inhouse CFD software FlowPro based on the discontinuous Galerkin method. Two following cases are considered. In the first case, the plates are fixed in time and the steady state is reached. The resulting velocity fields are com-
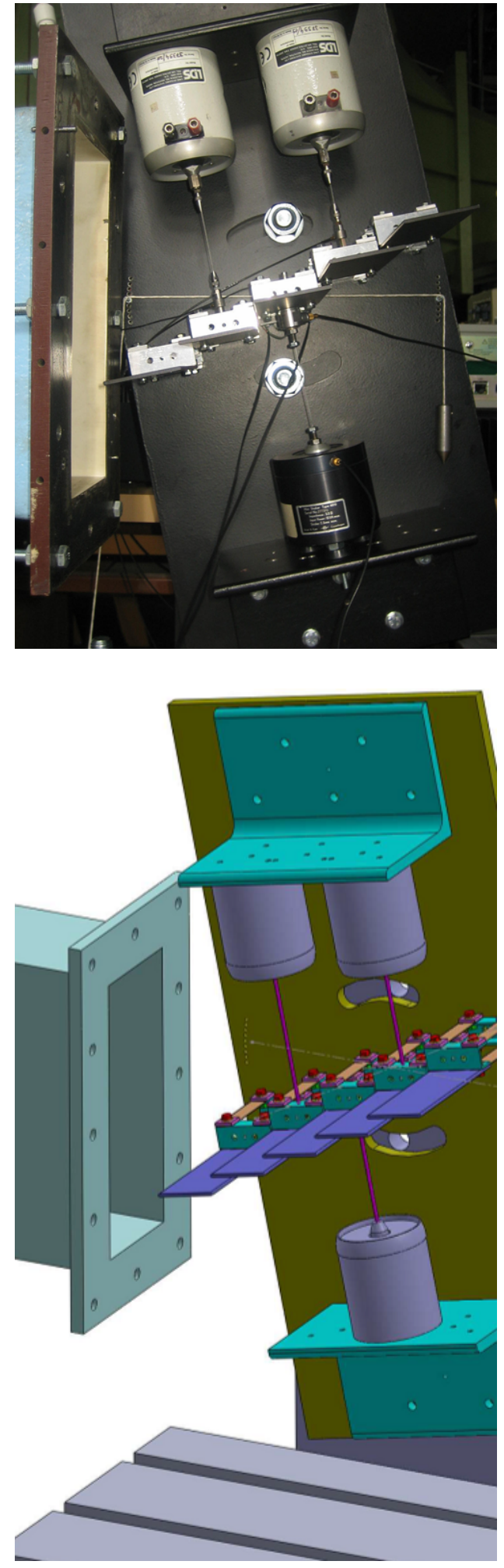

Fig. 2. Experimental stand of the simplified blade cascade (top) and its model created with CAD software (bottom).

pared. In the second case, the three middle flat plates perform kinematic harmonic motion. In both cases the same boundary condition are applied. The computation domain is shown in Figures 3 and 4. In order to obtain correct results, it turns out that it is necessary to include the inlet part of stand in the geometry as shown in Fig- 
ure 3. The inlet velocity magnitude is set to $u=40 \mathrm{~m} \mathrm{~s}^{-1}$, the angle of attack to $\alpha=11.4^{\circ}$ and inlet pressure to $p_{i}=101325 \mathrm{~Pa}$. The computed and measured velocity fields for steady plates are shown in Figures 5 and 6, respectively. One can see that the velocity fields have similar character. The values of the velocity magnitude are approximately the same in both cases.

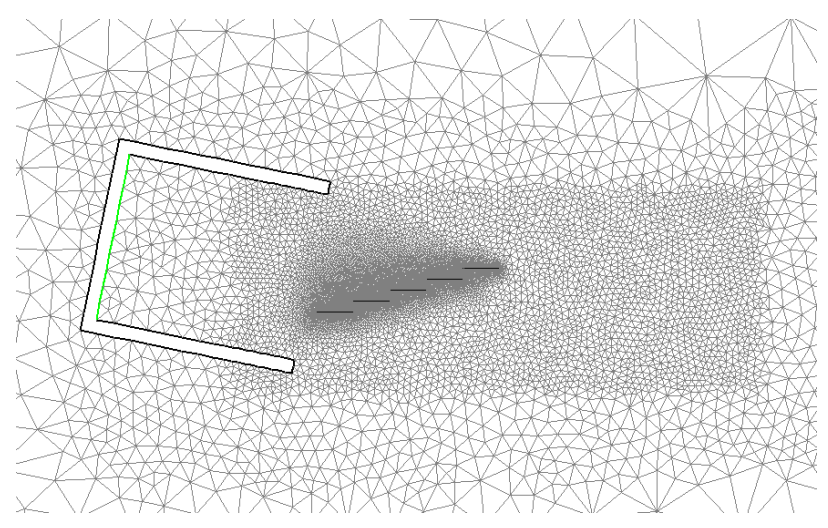

Fig. 3. Computational domain and triangular mesh.

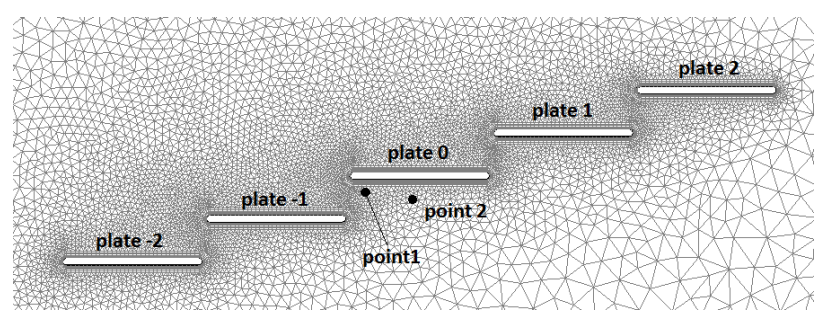

Fig. 4. Detail of computational mesh in the vicinity of simplified cascade.

In the second test case, the three middle plates perform kinematic harmonic motion in the y-direction $y=$ $A \sin (2 \pi f t+\phi)$ with an inter-blade phase angle $\phi=\pi / 2$ for the upper plate (plate 1), $\phi=0$ for the middle plate (plate 0 ) and $\phi=-\pi / 2$ for the bottom plate (plate -1 ), see Figure 4. The amplitude and frequency of the harmonic motion is $A=0.00283 \mathrm{~m}$ and $f=40 \mathrm{~Hz}$, respectively. In order to compare the unsteady velocity fields two points between plates 0 and -1 were chosen as shown in Figure 4. One period of the oscillating velocity at point 1 and 2 is plotted in Figure 7 and Figure 8, respectively. A reasonable agreement between numerical and experimental data is apparent at point 1 , on the other hand the difference in amplitude is significant at point 2. Finally, Figures 9 and 10 show power spectral density of $y$ and $x$ component of velocity corresponding to the frequency of $40 \mathrm{~Hz}$ obtained by the numerical simulation and Figures 12 and 13 also show power spectral density of $y$ and $x$ component of velocity this time obtained from the experiment. Note that the experimental data are not defined in front of the flat plates because of their shade with respect to the laser beams. In addition, the computed power spectral density of pressure is shown in Figure 11. A qualitative comparison of results reveal a good agreement.

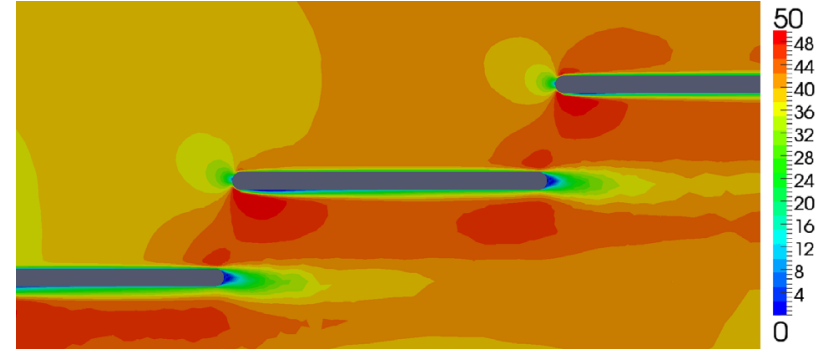

Fig. 5. Computed steady velocity field

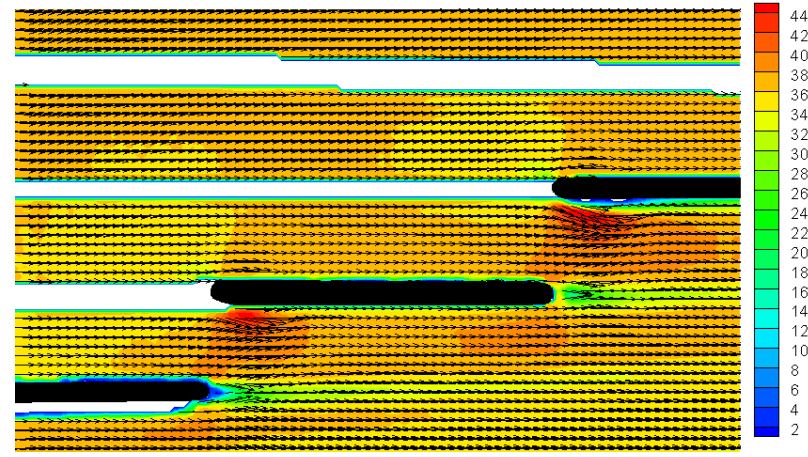

Fig. 6. PIV measured steady velocity field

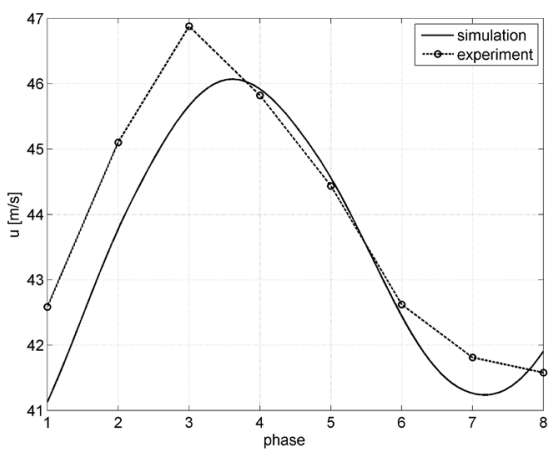

Fig. 7. Comparison of $x$ component of velocity at point 1

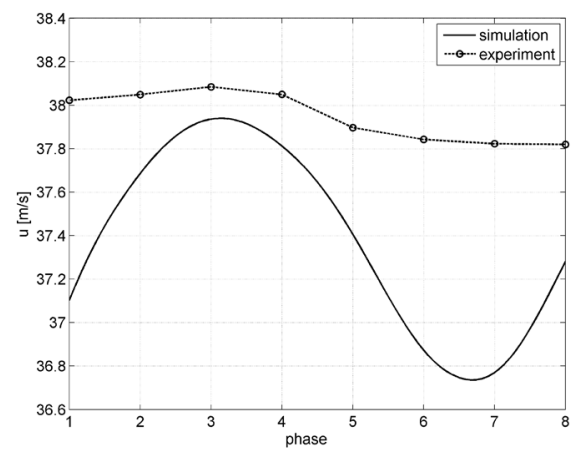

Fig. 8. Comparison of $x$ component of velocity at point 2

\section{Conclusion}

The comparison between numerical and experimental data show a reasonably good agreement. Hence, the in-house 
CFD solver based on the discontinuous Galerkin method is applicable for solution of blade cascade problems. The results of power spectral densities shows the higher values between the tip of the plate and the end of the bottom plate, see Figures 9, 11, 12, 13. This higher values represents aerodynamic coupling between plates related to oscillation frequency $40 \mathrm{~Hz}$. The use of higher oscillation frequency will probably lead to higher aerodynamic coupling between the plates. This assumption will be studied in future work.

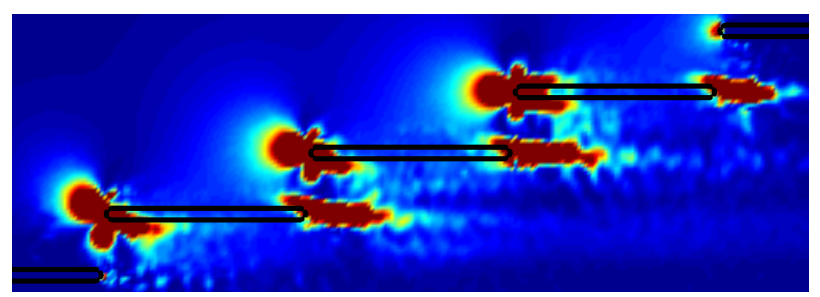

Fig. 9. Computed power spectral density of $y$-component of velocity

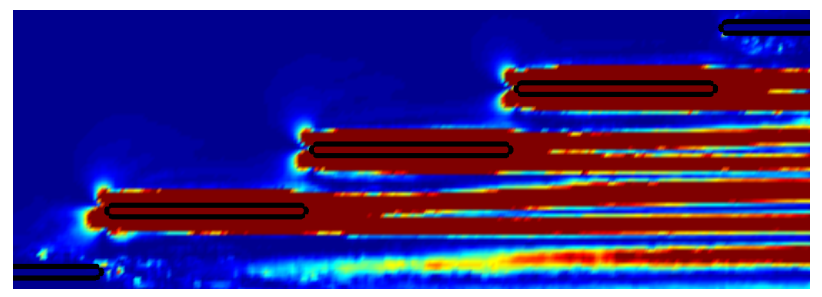

Fig. 10. Computed power spectral density of $x$-component of velocity

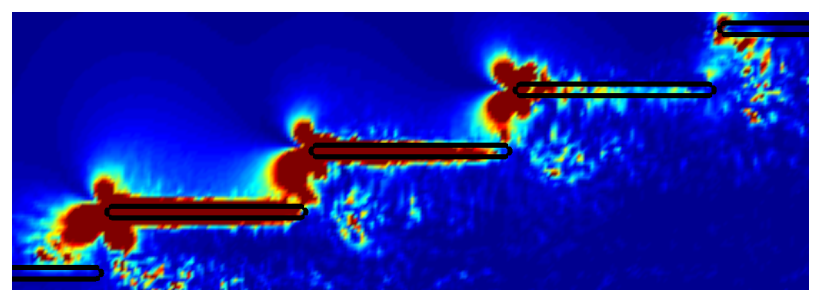

Fig. 11. Computed power spectral density of pressure

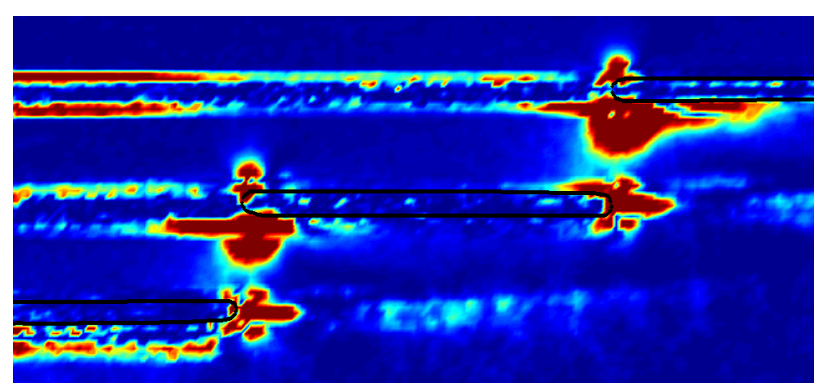

Fig. 12. Measured power spectral density of $y$-component of velocity

\section{Acknowledgements}

The authors appreciate the kind support by the grant GA 16-04546S "Aero-elastic couplings and dynamic behaviour of rotational periodic bodies" of the Czech Science Foundation and by the internal student grant projects SGS-2016-038 of the University of West Bohemia.

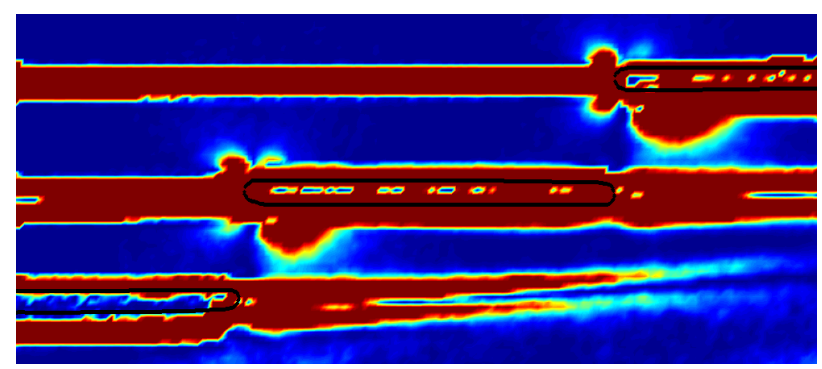

Fig. 13. Measured power spectral density of $x$-component of velocity

\section{References}

[1] H. M. Owens, W. E. Trumpler, ASME Paper, 49-A-64 (1949)

[2] E. K. Armstrong, R. E. Stevenson, J. R. Aero. Soc., 64 (591), 117-130 (1960)

[3] D. Schlafli, Experimentelle untersuchung der instationaren stromung in oscillierenden ringgittern. $\mathrm{PhD}$ Thesis No. 800, Lausanne (1989)

[4] J. Vimmr, O. Bublík, A. Pecka, L. Pešek, In Proceedings 6th ECCOMAS Thematic Conference on Computational Methods in Structural Dynamics and Earthquake Engineering (COMPDYN 2017), Rhodes Island, Greece, (2017)

[5] M. Feistauer, J. Česenek, NM\&A, 60 (46), 1-13 (2011)

[6] P. R. Spalart, S. R. Allmaras, AIAA Paper, 92-439 (1992)

[7] W. H. Reed, T. R. Hill, Los Alamos Scientific Laboratory, Report No. LA-UR-73-479 (1973)

[8] B. Cockburn, C.-W. Shu, J. Sci. Comput., 16 (3), 173261 (2001)

[9] O. Bublík, J. Vimmr, A. Jonášová, Appl. Math. Comput., 267, 329-340 (2015)

[10] D. J. Kirshman, F. Liu, Comput. Fluids, 35 (6), 571586, (2006)

[11] J. Fürst, K. Kozel, P. Furmánek, Kybernetika, 45 (4), 567-579 (2009) 